\title{
Acquisition des liaisons nominales et verbales : de la lexicalisation à l'abstraction des constructions.
}

\section{The Acquisition of Nominal and Verbal Liaisons: From Lexicalized to Abstract Constructions.}

\author{
Loïc Liégeois \\ Université d'Orléans, Laboratoire Ligérien de Linguistique
}

\section{Résumé}

Cet article a pour objectif de proposer une première analyse de l'acquisition de la liaison verbale par l'enfant francophone prélecteur. Dans ce but, nous présentons une étude fondée sur un corpus d'interactions entre trois jeunes enfants et leurs parents. En prenant comme cadre les Grammaires de Construction et les Modèles Basés sur l'Usage, nos analyses montrent que, comme la liaison nominale, l'acquisition de la liaison verbale semble suivre un processus menant de la mémorisation de constructions lexicalisées à une abstraction fondée sur les analogies et conditionnée par des facteurs liés à l'usage.

Mots-clés : acquisition ; liaison ; abstraction ; effets d'input ; corpus denses

This paper deals with the problematic of verbal liaison acquisition by young French Children. The main goal is to propose a study based on corpora of natural interactions between three children and their parents. Taking Construction Grammars and Usage-Based Models as theoretical frameworks, our results show that verbal liaison acquisition, like nominal liaison acquisition, is a process leading to abstraction based on analogies from frozen constructions. The process seems to be largely conditioned by usage factors.

Keywords: acquisition ; liaison ; abstraction ; input effects ; dense corpora

\section{INTRODUCTION}

Réaliser une liaison consiste à produire une consonne (CL) entre deux mots : un Mot1 et un Mot2, comme entre un et ours par exemple (un n-ours). Deux grandes catégories de liaison sont généralement distinguées : les liaisons variables, facultativement réalisées en fonction de critères principalement discursifs et sociolinguistiques et les liaisons catégoriques, toujours réalisées. Notre étude portera sur cette dernière catégorie.

Si le processus d'acquisition de la liaison nominale est bien décrit (Chevrot et al., 2013 ; Wauquier \& Shoemaker, 2013), l'acquisition de la liaison verbale a été mise de côté pour deux raisons. Tout d'abord, les chercheurs se sont logiquement focalisés sur le contexte générant le plus d'erreurs dans les productions enfantines. S'appuyant sur un relevé au vol d'erreurs dans les productions d'une fillette entre 2 et 6 ans, Dugua (2006) note que $85 \%$ des erreurs se produisent entre un déterminant et un nom contre $5,5 \%$ avant une forme verbale. De plus, les liaisons verbales sont difficiles à faire produire à de jeunes sujets en situation expérimentale. Dugua et al. (2017) notent par exemple qu'au cours d'une tâche de dénominations d'images visant à faire produire des liaisons verbales, seulement $23 \%$ des sujets de 3 à 6 ans produisent l'énoncé cible. Notre étude propose, pour la première fois, une analyse spécifique de l'acquisition de la liaison verbale fondée sur un corpus écologique. À notre connaissance, seule l'étude de Dugua et ses collègues (2017) s'est auparavant focalisée sur l'acquisition de ce type de liaison, en s'appuyant sur des données provoquées au cours de tâches expérimentales.

L'objectif de cette recherche est double. Tout d'abord, nous souhaitons proposer une analyse unifiée des Grammaires de Construction (GC) et des Modèles Basés sur l'Usage (MBU) en mettant en relief ce qui relie les deux cadres. Nous suivons Leroy et al. (2009) en regroupant les deux modèles sous le terme de Théorie Usage et Construction (TUC) et nous illustrerons la façon dont ce cadre a été utilisé pour rendre compte de l'acquisition de la liaison nominale. Ensuite, nous nous appuierons sur les concepts de la TUC dans le but de proposer une première analyse de l'acquisition de la liaison verbale. Adoptant dans un premier temps une démarche heuristique visant à décrire les usages enfantins, notre objectif sera ensuite de vérifier si le scénario basé sur l'usage proposé pour l'acquisition de la liaison nominale (Chevrot et al., 2013) est extensible aux contextes de liaisons verbales. Plus précisément, nous mettrons en rapport les productions enfantines avec les caractéristiques du discours parental qui leur est adressé dans le but de vérifier les hypothèses émises par la TUC.

\section{USAGE, CONSTRUCTION ET ACQUISITION DE LA LIAISON NOMINALE}

\subsection{Grammaires de constructions et Modèles basés sur l'usage : des objectifs différents...}

Les $\mathrm{GC}^{1}$ et les $\mathrm{MBU}$ entretiennent une relation étroite, en raison notamment de leur émergence à l'intérieur du champ des approches dites fonctionnelles et de leur développement en relation avec la linguistique et la grammaire cognitives. Si les deux

\footnotetext{
${ }^{1}$ On distingue plusieurs variantes des grammaires de construction (François, 2011 ; Goldberg, 2006 ; Gries, 2013), dont la grammaire de construction à base cognitive (Goldberg, 2006) que nous prenons comme référence ici car c'est cette variante qui a comme principal centre d'intérêt les phénomènes d'acquisition (Diessel, 2013). Il n'en demeure pas moins que toutes les approches constructionnelles ont en commun les principes de base que nous définissons en parlant des Grammaires de
} 
Author's Pre-print: Liégeois, L. (2017). Acquisition des liaisons nominales et verbales : de la lexicalisation à l'abstraction des constructions. Langue Française, 194(2). pp. 125-146. doi:10.3917/lf.194.0125.

cadres sont souvent confondus et combinés dans la littérature, c'est qu'ils se sont enrichis l'un l'autre au cours des trois dernières décennies (Leroy et al., 2009). De ce fait, l'un peut difficilement être abordé sans prendre l'autre en considération. En définissant les MBU, Diessel (2013) affirme par exemple que les études sur le développement grammatical réalisées dans ce cadre ont été largement influencées par les GC :

[...] usage-based researchers have developed a very different framework for the analysis of grammatical development that is crucially influenced by assumptions of construction grammar. (Diessel, $2013: 348$ )

Quant aux GC, elles ont trouvé dans les MBU un scénario développemental crédible. La relation entre les deux cadres théoriques est illustrée par Goldberg affirmant que "most construction grammars these days are usage-based" (2006:14). Les frontières séparant les deux cadres sont extrêmement floues, les GC et les MBU partageant les mêmes postulats de base. Seuls leurs objets d'attention diffèrent. Ainsi, l'objectif des GC est de décrire comment la connaissance grammaticale est organisée en mémoire alors que celui des $\mathrm{MBU}$, comme l'affirme Bybee, est de déterminer par quels procédés cognitifs un locuteur accède à cette dernière :

While all linguists are likely to agree that grammar is the cognitive organization of language, a usage-based theorist would make the more specific proposal that grammar is the cognitive organization of one's experience with language. (Bybee, $2006: 711$ )

Nous suivons ainsi Dugua en postulant que « les grammaires de construction permettent de réfléchir aux modalités de traitement et d'interaction des niveaux de la connaissance linguistique et les théories basées sur l'usage aux processus mis en œuvre pour accéder à cette connaissance » $(2006: 86)$.

\section{2. ...mais des postulats communs.}

Si leurs objets d'étude diffèrent, GC et MBU partagent un ensemble de postulats dont les principes se trouvent au fondement de la linguistique cognitive (Langacker, 1986, 1987). Parmi ces postulats, nous trouvons notamment la remise en cause de l'autonomie de la compétence linguistique vis-à-vis des autres compétences cognitives. Concernant l'acquisition du langage, cette remise en cause est essentielle et marque une rupture nette avec les conceptions innéistes et universalistes des approches génératives formelles. La grammaire d'un locuteur, plutôt que de correspondre à un inventaire de règles, est représentée par un ensemble de structures plus ou moins larges et abstraites, souvent appelées constructions (Goldberg, 1995). Les GC et les MBU rejettent donc l'idée d'une organisation grammaticale scindée en deux parties : d'un côté les règles syntaxiques, de l'autre le lexique regroupant les formes sur lesquelles ces règles s'appliquent (Pinker, 1999). Ce postulat, symbolisé par ce que Langacker a nommé "rule/list fallacy" (1987) et illustré par la citation infra, est un point de désaccord central entre approches génératives formelles et basées sur l'usage :

$[\mathrm{I}] \mathrm{t}$ does not strive to reduce language to as abstract a rule system as possible, because particular (lexically specific) and abstract phenomena are the same in kind, namely symbolic form-function units. (Behrens, 2009 : 385)

Les constructions sont symboliques dans le sens où elles supportent des indices sémantiques, situationnels et interactionnels (Goldberg, 1995 ; Tomasello, 2003). Ce point est le troisième permettant de caractériser communément les GC et les MBU. Dans ce cadre, la signification d'une structure peut être soit dérivée des sous-structures qui la composent, soit directement mémorisée avec la structure elle-même (Goldberg, 2006 ; Langacker, 1987).

Les liens étroits unissant GC et MBU nous amènent, comme Leroy et al. (2009), à proposer une analyse unifiée des deux cadres et à les réunir sous le terme Théorie Usage et Construction (TUC). Après avoir présenté les MBU et les GC en exposant les postulats qu'ils partagent, nous allons nous concentrer sur la définition des grandes notions qui constituent les clefs de voûte de la TUC. Loin de procéder à une revue exhaustive des acceptions de ces notions, notre objectif est de mettre l'accent sur les concepts qui seront investis au cours de nos analyses.

\subsection{La Théorie Usage et Construction en quatre concepts clef}

\subsubsection{Construction}

Dans le cadre de la TUC, une construction peut correspondre à des « formes » et des « tailles » différentes. Ainsi, un morphème ou un mot peuvent être mémorisés au même titre qu'un schéma complètement abstrait (cf. Tableau 1). En ce qui concerne la taille, une construction lexicalisée peut correspondre à un mot graphique ou à une expression idiomatique lexicalisée. Ce postulat de la TUC permet de se différencier des approches formelles sur deux points (Tomasello, 2003) :

- le plus haut niveau d'abstraction repéré par le linguiste n'est pas nécessairement celui qui est intériorisé par le locuteur ;

les expressions figées, les idiomes, font partie intégrante de la grammaire du locuteur.

Tableau $1:$ Exemples de constructions. Adapté de Goldberg $(2006: 5)$.

\begin{tabular}{|l|l|}
\hline \multicolumn{2}{|l|}{ Exemples de constructions, variant en taille et en complexité } \\
\hline Appellation classique & Exemple $^{2}$ \\
\hline Morphème & $\mid$ re-|,|-ette $\mid$ \\
\hline Mot & $\mid$ fenêtre $\mid$ \\
\hline Mot complexe lexicalisé & $\mid$ casse-noisette $\mid$ \\
\hline Expression lexicalisée & $\mid$ qui va à la chasse perd sa place $\mid$ \\
\hline
\end{tabular}

Construction. Pour plus de détails sur les différences entre les variantes des GC, voir notamment François (2008:15) et Goldberg (2006 : 206-226).

${ }^{2}$ Nous serons amenés à plusieurs reprises à illustrer nos propos en présentant des exemples de constructions. Une construction sera encadrée par deux barres verticales (II). Les catégories grammaticales (nom, verbe) seront représentées la plupart du temps par leur initiale $(\mathrm{N}, \mathrm{V})$. Les éléments lexicalisés seront renseignés en italique, tandis que les propriétés phonétiques seront indiquées entre crochets (comme [Consonne]-N par exemple pour indiquer qu'il s'agit d'un nom débutant par une consonne). Les slots, ou emplacements vides, seront quant à eux indiqués par la lettre $\mathrm{X}$ et les fonctions grammaticales en exposants. 
Author's Pre-print: Liégeois, L. (2017). Acquisition des liaisons nominales et verbales : de la lexicalisation à l'abstraction des constructions. Langue Française, 194(2). pp. 125-146. doi:10.3917/lf.194.0125.

\begin{tabular}{|l|l|}
\hline Expression partiellement lexicalisée & $\mid$ se moquer de $+\mathrm{X} \mid$ \\
\hline Construction conditionnelle & $\mid$ si $\mathrm{X}$ je $\left.\mathrm{X}\right|_{\text {CONDITION }}$ \\
\hline Construction transitive double & $\left|\mathrm{X}_{\mathrm{SUJET}}+\mathrm{V}+\mathrm{X}_{\mathrm{OBJET}}+\mathrm{X}_{\mathrm{OBJET}}\right|$ (il donne un bonbon à son fils) \\
\hline Construction passive & $\mid \mathrm{X}_{\mathrm{SUJET}}+\mathrm{Aux} .+\mathrm{PP}+$ par $+\mathrm{X} \mid$ \\
\hline
\end{tabular}

L'ensemble de ces constructions constitue la grammaire d'un locuteur que Langacker définit comme "a structured inventory of conventional linguistic units" (2008:222). L'une des caractéristiques principales de ces unités est qu'elles supportent les indices sémantiques et pragmatiques extraits des situations d'interaction au cours desquelles elles sont produites. Comme nous l'avons vu précédemment, il s'agit d'un point d'accord central entre les GC et les MBU, comme en témoignent ces affirmations de Bybee et Goldberg :

The crucial idea behind the construction is that it is a direct form-meaning pairing that has sequential structure and may include positions that are fixed as well as positions that are open. (Bybee, 2010:9)

All levels of grammatical analysis involve constructions: learned pairings of form with semantic or discourse function, including morphemes or words, idioms, partially lexically filled and fully general phrasal patterns. (Goldberg, $2006: 5$ )

En un sens, la TUC place donc au même niveau des unités qui reçoivent des traitements particuliers de la part des approches considérant la grammaire comme un ensemble à deux niveaux (règles syntaxiques et lexique). Avec la TUC, cette distinction est rompue et remplacée par un continuum, comme postulée dans les grammaires cognitives. Ce continuum est borné d'un côté par des expressions lexicalisées et de l'autre par des schémas abstraits composés de slots (emplacements ouverts) pouvant accueillir des éléments correspondant à leurs catégorisations sémantiques et/ou grammaticales :

The open slots in constructions are subject to categorization in terms of semantic features (such as 'motion verb') or grammatical features (such as pronoun). (Bybee, $2001: 343$ )

Comme l'affirment Bybee et Hopper (2001), puisqu'aucun locuteur ne parle en utilisant des mots ou des morphèmes isolés, il est logique que les constructions stockées soient la plupart du temps composées de plusieurs morphèmes ou plusieurs mots. Cependant cet ensemble ne correspond pas à un simple inventaire de structures car les constructions, supportant des propriétés phonologiques, syntaxiques et sémantiques, sont reliées et organisées entre elles en réseaux au sein de ce qui a souvent été défini comme un "constructicon" (Goldberg, 2006).

\subsection{2. Évènements d'usage}

En mettant l'accent sur le terme d'usage, la TUC rompt nettement avec les dichotomies compétence/performance et langue/parole. En effet, les MBU considèrent que la connaissance de la langue découle des évènements d'usage auxquels le locuteur est exposé : "the speaker's linguistic system is fundamentally grounded in "usage events"” (Kemmer \& Barlow, 2000 : viii).

Cependant, les évènements d'usage ne doivent pas être réduits à l'ensemble des énoncés entendus par un locuteur. Un évènement d'usage représente une structure plus complexe comprenant l'ensemble des informations qui permettent d'interpréter ou de produire un énoncé linguistique et supporte ainsi les indices propres à la situation de communication participant à son interprétation. L'axe pragmatique est donc un moteur de la construction de la grammaire (Tomasello, 2003). Toutefois la compétence linguistique n'est pas définitivement fixée au sortir de la période d'acquisition puisque celle-ci est sans cesse en confrontation avec des évènements d'usage qui la modifient constamment (Bybee, 2006). Cette particularité soulève le double rôle des évènements d'usage qui « résultent du système linguistique lui-même mais le façonnent également par une sorte de boucle rétroactive » (Nardy, $2008:$ 74).

\subsubsection{Fréquence}

Il est logique que, dans un cadre théorique affirmant que la connaissance de la langue émerge de son usage, la notion de fréquence soit centrale. Dans l'introduction à l'ouvrage qu'ils ont coordonné, Kemmer et Barlow (2000) présentent les neuf grands principes au fondement des MBU. La notion de fréquence arrive en deuxième position, juste après la présentation de la notion d'usage, reflétant son importance dans la définition du modèle. Les premiers mots ouvrant la section sur la fréquence sont d'ailleurs sans équivoque :

Because the system is largely an experience-driven one, frequency of instances is a prime factor in its structure and operation. (Kemmer \& Barlow, $2000: \mathrm{x}$ ).

La fréquence apparaît pour la TUC comme le principal moteur de la structuration de la compétence linguistique. Cette notion prend encore plus de sens au cours de la période d'acquisition, le scénario basé sur l'usage de l'acquisition du langage ayant mis en évidence son rôle primordial (Tomasello, 2003) et de nombreuses études ayant relevé des effets de fréquence en input sur l'acquisition lexicale, grammaticale ou phonologique (Liégeois, $2014: 35-65$ ).

Deux types de fréquence linguistique sont souvent distingués en raison de leurs rôles dans la structuration et l'organisation des constructions (Goldberg, 2006 ; Langacker, 2009 ; Tomasello, 2003 ; Abbot-Smith \& Tomasello, 2006 ; Bybee, 2006,2010 ; Croft \& Cruse, 2004 ; Matthews et al., 2005) : la fréquence des formes (tokens) et la fréquence des types. Ces deux faits de fréquence auraient des répercussions différentes sur l'ancrage cognitif (entrenchment), l'abstraction et la productivité des constructions (Behrens, 2009 ; Bybee, 2006 ; Tomasello, 2003). Selon Langacker, la fréquence d'un type de construction favorise l'abstraction (et donc sa productivité) d'une construction alors que la fréquence d'une forme favorise l'ancrage cognitif d'une forme lexicalisée (2009 : 638) : " [...] token frequency helps preserve idiosyncratic forms, whereas type frequency contributes to a pattern's productivity".

Cependant, la formation de schémas abstraits n'efface pas nécessairement la trace de la construction lexicalisée préalablement mémorisée. En effet, les GC comme les MBU considèrent que la mémoire humaine est capable de stocker des informations redondantes structurées à différents niveaux (Kemmer \& Barlow, 2000 : IX), c'est-à-dire différents exemplaires.

\subsubsection{Exemplaire}

Alors que la notion d'exemplaire apparait complexe et possède plusieurs acceptions, nous nous concentrerons sur la manière dont cette notion est devenue un concept clé de la TUC : 
Author's Pre-print: Liégeois, L. (2017). Acquisition des liaisons nominales et verbales : de la lexicalisation à l'abstraction des constructions. Langue Française, 194(2). pp. 125-146. doi:10.3917/lf.194.0125.

$[\ldots]$ exemplar theory $[\ldots]$ assumes that a category is "represented in memory by a large cloud of remembered tokens of that category" (Pierrehumbert $2001: 140$ ), and that new instances are shaped or interpreted on the basis of clusters of stored instances. I have no basic disagreement with exemplar theory; I take it as being essentially correct. I do not however consider it to be an actual alternative, but rather a particular implementation of the usage-based approach. (Langacker, 2009: 636)

Plusieurs points communs à la théorie des exemplaires et à la TUC peuvent être soulevés, comme par exemple le postulat d'une mémorisation de la forme accompagnée de l'ensemble des informations phonétiques et contextuelles concernant sa production (Bybee, 2010 ; Pierrehumbert, 2001). La TUC reconnait le stockage, sous forme d'exemplaires, de constructions lexicalisées à partir desquelles des constructions plus abstraites émergent. Le processus d'abstraction postulé par les tenants des GC et le stockage holistique des variantes proposé par la théorie des exemplaires se retrouvent donc au sein des MBU, d'ailleurs parfois définis comme des modèles hybrides "in which much of the extraneous details of original instances are retained but where some kind of more abstract schema is gradually formed on the basis of these" (Abbot-Smith \& Tomasello, 2006 : 282). Cette hybridation est particulièrement perceptible dans les propos de Kemmer \& Barlow affirmant qu'une construction lexicalisée peut représenter un exemplaire d'une construction abstraite également mémorisée.

[...] the mind can potentially represent the same structure in multiple ways, and hence the grammar includes both specific items and the more general patterns they are instances of. (Kemmer \& Barlow, 2000 : IX).

\subsection{Acquisition de la liaison nominale dans le cadre de la TUC : de la lexicalisation à l'abstraction}

Essentiellement fondés sur des données expérimentales, les travaux sur l'acquisition de la liaison nominale ont mené à la proposition de deux scénarios développementaux directement influencés par le débat sur la représentation lexicale de la CL chez l'adulte (Côté, 2005). Si ces deux scénarios s'accordent sur la chronologie développementale et la typologie des erreurs enfantines, ils s'opposent au niveau de leur interprétation.

Le scénario phonologique (Wauquier \& Shoemaker, 2013 ; Wauquier, 2009 ; Wauquier-Gravelines \& Braud, 2005) analyse le processus d'acquisition de la liaison nominale comme un phénomène fondé sur des principes abstraits. S'appuyant sur le cadre de la phonologie plurilinéaire appliquée à la liaison (Encrevé, 1988), la CL est définie dans ce scénario comme un segment doublement flottant. Les erreurs relevées dans les productions enfantines seraient le reflet de la mise en place progressive de ce statut particulier et ne seraient pas conditionnées par des facteurs liés à l'usage. Le processus d'acquisition de la liaison nominale est défini comme résultant de la mise en place de principes généraux, universels et abstraits (comme le " bootstrapping morpho-phonologique » par exemple) et la CL est décrite comme lexicalement encodée à la finale du Mot1 (Wauquier \& Shoemaker, 2013).

Le scénario lexical (Bybee, 2001, 2005 ; Chevrot et al., 2005, 2007, 2009, 2013) se fonde quant à lui sur les quatre grandes notions de la TUC décrites précédemment et sur le scénario de l'acquisition du langage décrit par Tomasello (2003). L'acquisition de la liaison nominale est définie comme un processus en trois stades au cours desquels les unités linguistiques, tout d'abord mémorisées sous la forme de constructions lexicalisées, vont progressivement s'abstraire sous l'effet des caractéristiques du bain linguistique.

\subsubsection{Premier stade du scénario lexical : mémorisation de constructions lexicalisées}

Le premier stade concerne la période pendant laquelle les enfants ne font aucune erreur en contexte de liaison, jusqu'à deux ans environ. Le modèle phonologique et le modèle lexical s'accordent sur ce stade : l'enfant mémoriserait une unité lexicale générale, nommée construction lexicalisée dans le cadre de la TUC. Cette construction serait composée du déterminant, du nom et de la CL qui les relie. Ainsi, si aucune erreur enfantine n'est relevée à ce stade, c'est parce que la CL est directement intégrée à la construction mémorisée, comme dans $\mid$ un n-ours $\mid$ par exemple. À ce moment de l'acquisition, l'enfant est donc capable de mémoriser plusieurs constructions figées différentes pour un même Mot2 : |un n-ours $|$,$| des z-ours \mid$ et $\mid$ un petit t-ours $\mid$ par exemple.

\subsubsection{Deuxième stade du scénario lexical : segmentation des constructions lexicalisées}

Le deuxième stade du scénario lexical verrait l'émergence de combinaisons Mot1-Mot2 rendues possibles au moyen de schémas abstraits fondés sur les items (Chevrot et al., 2009). Ces schémas se forment sous l'influence des régularités repérées par l'enfant entre les différentes constructions dans lesquelles une même forme est employée. En contexte de liaison, les régularités repérables par l'enfant sont à mettre en lien avec l'asymétrie des frontières lexicales et phonologiques. Par exemple, tous les Mots2 produits après le déterminant « un » vont débuter par une consonne (la consonne initiale du Mot2 si celui-ci débute par une consonne ou la $\mathrm{CL}[\mathrm{n}]$ si le Mot2 est à initiale vocalique). La régularité repérée par l'enfant est donc que les constructions de type $|\mathrm{DET}+\mathrm{N}|$ se composent :

d'un élément fixe, par exemple le déterminant « un»

et d'un second élément débutant par une consonne.

Sur cette base, les constructions préalablement mémorisées sous la forme de constructions lexicalisées, comme $\mid$ un n-ours|, vont être segmentées en deux constructions distinctes. D'un côté, l'enfant mémorisera des constructions abstraites, du type $\mid u n+[$ Consonne]-X|. D'un autre, procédant à une segmentation sur la base du schéma standard de la syllabe en français (ConsonneVoyelle), l'enfant mémorisera le Mot2 avec à son initiale la CL (par exemple $\mid n$-ours $\mid$ ). Ainsi, plusieurs exemplaires d'un même Mot2, comme $\mid n$-ours $||$,$z -ours \mid$ et $\mid t$-ours $\mid$, vont pouvoir être mémorisés à partir des énoncés « un ours », « des ours » et « petit ours ».

Dans le cadre de la TUC, les erreurs de remplacement de la CL comme « un z-ours », relativement fréquentes à ce stade (cf. Figure 4), résulteraient de l'insertion du mauvais exemplaire de Mot2 (par exemple $\mid n$-ours $\mid$ ) dans le slot d'une construction plus abstraite comme $\mid$ des $+[$ Consonne $]-\mathrm{X} \mid$.

\subsubsection{Troisième stade du scénario lexical : renforcement des liens entre constructions abstraites et exemplaire correct de Mot 2}

$\mathrm{Au}$ cours du troisième stade du scénario, les erreurs en contexte nominal tendent à diminuer jusqu'à disparaître. Selon le scénario lexical, l'enfant développerait un schéma plus abstrait sur la base des collocations entre un Mot1 spécifique et une série de Mots2 de plus en plus variés. La diversification des types de constructions en contexte $|u n+\mathrm{N}|$ amènerait par exemple l'enfant à généraliser la relation entre le Mot1 «un» et les exemplaires de Mots2 débutant par la consonne /n/. L'enfant saisirait 
Author's Pre-print: Liégeois, L. (2017). Acquisition des liaisons nominales et verbales : de la lexicalisation à l'abstraction des constructions. Langue Française, 194(2). pp. 125-146. doi:10.3917/lf.194.0125.

progressivement que lorsqu'il possède plusieurs exemplaires d'un Mot2, celui rencontré après " un » débute toujours par/n/. Sous cet effet, la construction précédemment active de type $\mid u n+[$ Consonne]-X $\mid$ se spécifierait en une construction de type $|u n+[n]-X|$.

Le scénario développemental que nous venons de présenter est étayé par un nombre important de données expérimentales obtenues au moyen de tâches de dénomination d'images (Dugua, 2006; Nardy, 2008), de jugements d'acceptabilité (Nardy, 2008) ou de chronométrie mentale (Siccardi, 2015). De plus, il a récemment été conforté par l'analyse de données recueillies en situation écologique (Liégeois, 2014). Toutefois, il est restreint au seul contexte de liaison nominale. Concernant l'acquisition de la liaison verbale, deux hypothèses ont simplement été mentionnées : l'une considère que le scénario de l'acquisition de la liaison verbale serait identique (Chevrot et al., 2007), l'autre postule qu'il s'agirait des deux processus différents (Dugua, 2006). L'objectif de cette étude est d'apporter les premiers éléments de débat en nous appuyant sur les données écologiques du corpus ALIPE.

\section{ACQUISITION DE LA LIAISON VERBALE : PREMIERS APPORTS DU PROJET ALIPE}

\subsection{Présentation du corpus d'étude et des jeunes sujets}

Le corpus ALIPE (Liégeois et al., 2014) regroupe trois corpus denses recueillis en situation écologique. Trois enfants premiers nés de familles biparentales et monolingues ont été enregistrés à leur domicile sur deux ou trois temps espacés de plusieurs mois. Un enregistreur numérique a été confié à chacun des couples de parents avec pour seule consigne d'enregistrer l'enfant une heure par jour pendant une semaine lors de situations propices aux interactions (bain, jeux et repas par exemple). La couverture du corpus ALIPE et les principales informations sur les sujets sont résumés dans le tableau infra.

Tableau 2: Principaux renseignements sur le corpus ALIPE et les sujets enregistrés

\begin{tabular}{|l|l|l|l|l|}
\hline Sujet & Temps de recueil & Âge du sujet & Nb. de graphies transcrites & Durée des enregistrements \\
\hline \multirow{3}{*}{ Salomé } & $\mathrm{T} 1$ & $2 ; 4$ & 29788 & $5 \mathrm{~h}$ \\
\cline { 2 - 5 } & $\mathrm{T} 2$ & $3 ; 0$ & 26102 & $4 \mathrm{~h} 55$ \\
\hline \multirow{3}{*}{ Baptiste } & $\mathrm{T} 1$ & $2 ; 11$ & 20398 & $4 \mathrm{~h} 55$ \\
\cline { 2 - 5 } & $\mathrm{T} 2$ & $3 ; 6$ & 19989 & $4 \mathrm{~h} 24$ \\
\hline \multirow{4}{*}{ Prune } & $\mathrm{T} 1$ & $3 ; 4$ & 33568 & $5 \mathrm{~h}$ \\
\cline { 2 - 6 } & $\mathrm{T} 2$ & $4 ; 0$ & 10695 & $1 \mathrm{~h} 59$ \\
\cline { 2 - 6 } & $\mathrm{T} 3$ & $5 ; 4$ & 25491 & $4 \mathrm{~h} 03$ \\
\hline \multicolumn{2}{|l|}{ Total } & & 166031 & $30 \mathrm{~h} 16$ \\
\hline
\end{tabular}

Cette méthodologie de recueil comporte plusieurs avantages dont ceux de minimiser les biais liés à la présence d'un observateur et de permettre le recueil de données produites en situation naturelle dans des contextes de production variés. Ainsi, si le corpus ALIPE comporte majoritairement des interactions parent(s)-enfant, il contient également des monologues enfantins et des discussions entre adultes.

Environ dix heures d'enregistrements par famille ont été transcrites, annotées et structurées au format XML-TEI (Text Encoding Initiative) et CHAT (Codes for the Human Analysis of Transcripts). Ces deux formats de structuration apparaissent complémentaires (Liégeois, 2013). Alors que le premier offre une solution de structuration standard, extensible, pérenne et interopérable, le second rend possible l'analyse du corpus à l'aide du logiciel associé CLAN (Computerized Language ANalysis ; MacWhinney, 2000). Au total, nous disposons de 2.492 contextes de liaisons catégoriques réalisées, dont 790 issues des productions enfantines. L'hétérogénéité de niveaux de compétences linguistiques des jeunes sujets (cf. Figure 1) permet d'avoir un aperçu de l'usage des liaisons verbales à différentes étapes du développement. Au regard des valeurs de longueur moyenne d'énoncés (MLU) et de diversité lexicale (VOCD), il apparaît clairement que nous disposons de sujets aux niveaux de développement hétérogènes.

Figure $1:$ Indices de développement langagier des jeunes sujets de l'étude

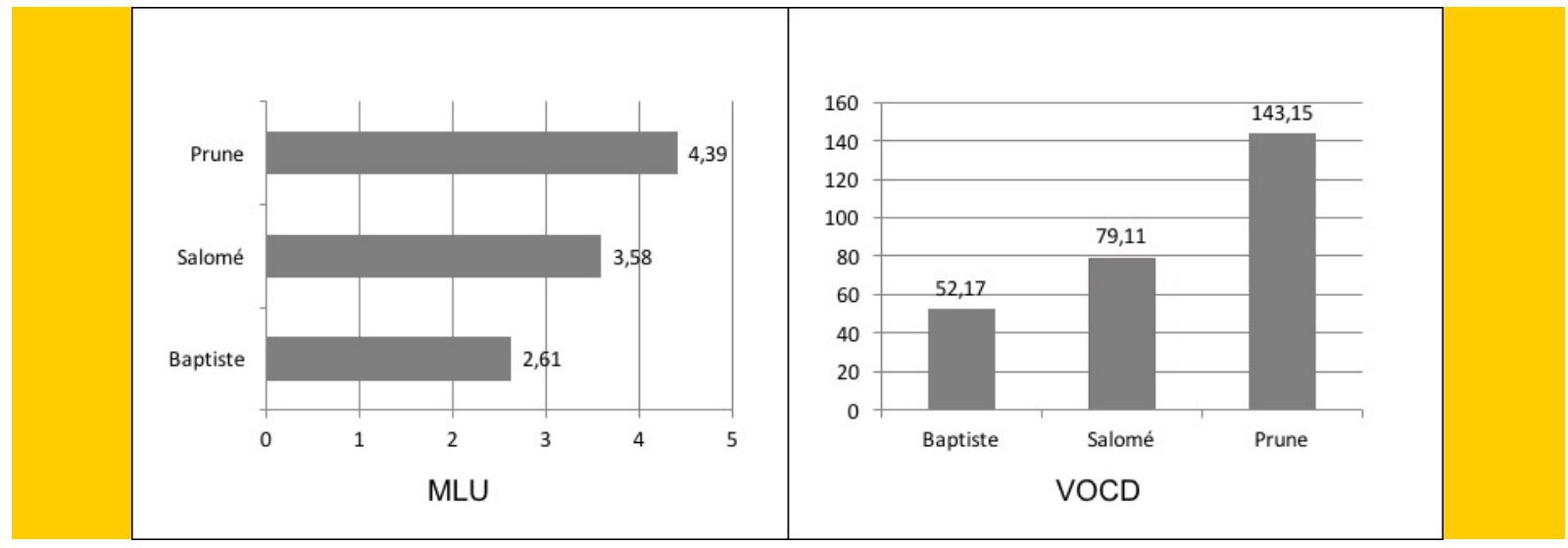

\subsection{Acquisition des liaisons nominales et verbales : un rapport asymétrique}

Comme le montre la figure 2, la division en trois stades du scénario lexical est perceptible à travers les taux de réalisations justes de la liaison nominale. En effet nous ne relevons aucune erreur chez notre sujet le moins avancé au premier temps de recueil. Pour rappel, ce stade est caractérisé, selon le scénario lexical, par la mémorisation de constructions lexicalisées contenant le déterminant, 
Author's Pre-print: Liégeois, L. (2017). Acquisition des liaisons nominales et verbales : de la lexicalisation à l'abstraction des constructions. Langue Française, 194(2). pp. 125-146. doi:10.3917/1f.194.0125.

le nom et la CL qui les relie. Le deuxième stade, illustré par les productions de Baptiste (au T2) et Salomé (au T1), voit le nombre d'erreurs augmenter. Le suivant, représenté dans notre corpus par les productions de Salomé (au T2) et de Prune, illustre la nette augmentation du taux de réalisations justes que le scénario lexical explique par la mise en place de constructions de plus en plus spécifiées restreignant les possibilités d'insertion des Mots2 au sein des schémas.

Figure 2: Taux de réalisations justes de la liaison nominale en fonction des trois stades du scénario lexical
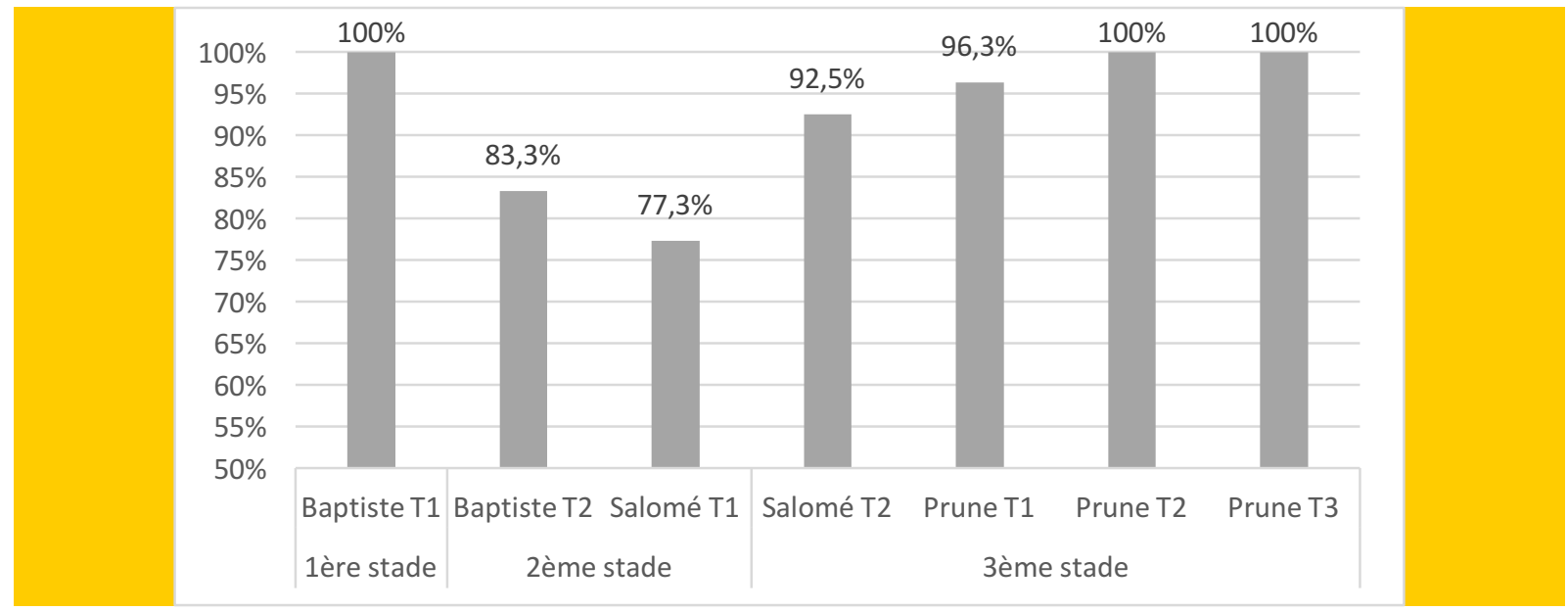

Afin de comparer l'acquisition de la liaison nominale et verbale, nous avons souhaité mettre en relation le taux de réalisations justes de la liaison nominale avec la proportion des erreurs impliquant un verbe. Comme le montre la figure 3 , il apparaît que cette relation est asymétrique : contrairement au taux de réalisations justes de la liaison nominale, la proportion des erreurs monopolisant un verbe augmente entre le stade 2 et 3. En effet, nous ne relevons aucune erreur en contexte verbal dans les productions de Baptiste et de Salomé au T1 (stades 1 et 2 du scénario). Les erreurs verbales émergent au cours du troisième stade, dans les productions de Salomé (au T2) et de Prune (7 erreurs comportant une forme verbale sur 65 au total ${ }^{3}$ ). L'asymétrie semble nette : les erreurs monopolisant une forme verbale apparaissent alors que la liaison catégorique nominale est maîtrisée. Cette asymétrie, observée à partir d'un nombre restreint de productions enfantines recueillies en usage, a récemment été confirmée par des données expérimentales recueillies auprès d'une large cohorte d'enfants (Dugua et al., 2017).

Figure 3 : Proportion d'erreurs monopolisant une forme verbale dans les productions enfantines

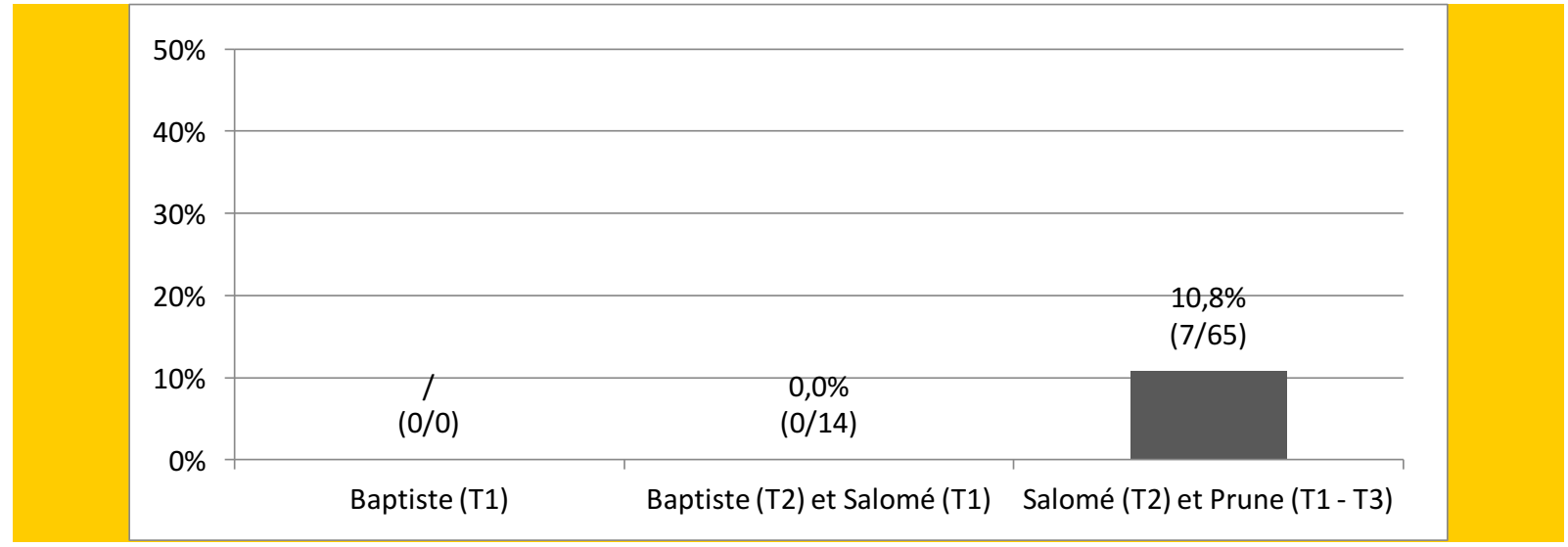

Une autre asymétrie peut être constatée au niveau de la typologie des erreurs relevées : alors que les remplacements de la CL en contexte $|\mathrm{DET}+\mathrm{N}|$ représentent près de $20 \%$ des erreurs enfantines (12/65), nous ne relevons aucun cas de remplacement en contexte $|\mathrm{PRO}+\mathrm{V}|$, mais uniquement des erreurs dues à une omission ou une adjonction de la CL (cf. Tableau 3).

Si le scénario proposé par Chevrot et al. (2009) pour l'acquisition de la liaison catégorique nominale peut s'appliquer à la liaison verbale, alors les asymétries observées devraient s'expliquer au regard des caractéristiques fréquentielles des liaisons verbales adressées à l'enfant. En considérant que la multiplication des erreurs en contexte de liaison nominale est due à l'abstraction et à la spécification progressive de constructions préalablement lexicalisées dans le constructicon enfantin, nous faisons alors l'hypothèse que l'apparition d'erreurs monopolisant un verbe à un stade plus avancé du développement résulte d'une abstraction plus tardive des constructions de type $|\mathrm{PRO}+\mathrm{V}|$. Nous faisons donc l'hypothèse que les constructions monopolisant une forme verbale restent plus longtemps mémorisées sous la forme de constructions lexicalisées dans le lexique enfantin. Cette proposition est renforcée par le fait

\footnotetext{
${ }^{3}$ Les autres erreurs ne sont pas commises en contexte de liaison entre un déterminant et un nom, mais majoritairement en contexte d'adjonction («ta z-orange ») et entre un adjectif et un nom (« un petit z-avion »).
} 
Author's Pre-print: Liégeois, L. (2017). Acquisition des liaisons nominales et verbales : de la lexicalisation à l'abstraction des constructions. Langue Française, 194(2). pp. 125-146. doi:10.3917/lf.194.0125.

que, contrairement au contexte $|\mathrm{DET}+\mathrm{N}|$, nous ne relevons aucune erreur en contexte $|\mathrm{PRO}+\mathrm{V}|$. Dans la suite, nous chercherons à vérifier cette hypothèse au regard des propriétés du discours adressé à nos jeunes sujets.

Tableau 3 : Relevé des erreurs enfantines monopolisant une forme verbale

\begin{tabular}{|l|l|l|}
\hline Enfant & Énoncé & Type d'erreur \\
\hline Salomé & tu n-en rachètes tu n-as racheté & \\
\hline \multirow{4}{*}{ Prune } & et les mammouths ne z-ont pas de zizi & \\
\cline { 2 - 2 } & c'est les ceux ceux qui z-ont des zizis & \multirow{3}{*}{ Adjonction } \\
\cline { 2 - 2 } & ceux qui z-ont pas & \\
\cline { 2 - 3 } & des amis qui z-étaient allés & \\
\cline { 2 - 3 } & il n-appuie sur un petit bouton & \\
\cline { 2 - 3 } & regardez je vous 0-offre & Omission \\
\hline
\end{tabular}

3.3. Émergence des erreurs en contexte de liaison verbale : une influence de l'input?

Pour la TUC, l'abstraction des constructions lexicalisées est conditionnée par la fréquence des formes et des types linguistiques en usage. À partir des données du corpus ALIPE, nous avons relevé l'ensemble des collocations Mot1-Mot2 produites en discours adressé à l'enfant ${ }^{4}$ (DAE). La distribution de ces collocations, dont les douze plus fréquentes en input sont présentées infra, illustre deux faits empiriques importants. Tout d'abord, un seul contexte nominal apparaît dans les douze cadres lexicaux de liaison les plus fréquents en input. Parmi les onze autres, nous relevons deux contextes de liaison appartenant à des constructions dites figées (comme "peut-être ») et neuf constructions verbales. De plus, alors que nous relevons près de 250 collocations Mot1-Mot2 différentes adressées aux enfants, les six cadres lexicaux de liaison les plus fréquents entre un pronom et un verbe ${ }^{5}$ représentent à eux seuls près de $21 \%$ des 1.209 liaisons produites en DAE.

Si les collocations du type $|\mathrm{PRO}+\mathrm{V}|$ sont celles qui apparaissent le plus fréquemment en input, ces collocations sont également davantage figées, dans le sens où un nombre très limité de verbes sont combinés avec un pronom en DAE. Dans une précédente étude (Liégeois, 2014), nous avons en effet cherché à calculer la diversité lexicale en contextes $|\mathrm{DET}+\mathrm{N}|$ et $|\mathrm{PRO}+\mathrm{V}|$ dans le DAE en nous appuyant sur la méthodologie mise en place par Chevrot et al. (2007). Cette méthode vise à obtenir un indice de variation lexicale en divisant le nombre total d'apparition d'un Mot1 par le nombre de formes différentes de Mot2 qui le suivent ${ }^{6}$.

En appliquant cette méthodologie, nous avons ainsi calculé l'indice de diversité lexicale pour l'ensemble des Mots1 produits au moins vingt fois par les parents de Prune et de Salomé. La distribution des Mots1 étudiés est indiquée dans le tableau 5 et montre une tendance nette : alors que les déterminants se combinent à un nombre important de Mots2 différents, les pronoms précèdent un nombre restreint de verbes.

Ce double effet (fréquence et figement des collocations verbales) favoriserait la mémorisation par l'enfant de constructions lexicalisées. Cette distribution plus restreinte des Mots2 en contexte verbal pourrait en partie expliquer pourquoi les erreurs sont plus nombreuses et précoces en contexte nominal. Nous pouvons en effet postuler que cette redondance des collocations Mot1-Mot2 en contexte verbal entraîne une segmentation plus tardive des constructions verbales lexicalisées par l'enfant, ce dernier ayant à sa disposition moins d'informations sur lesquelles s'appuyer pour procéder aux abstractions fondées sur les analogies.

Tableau 4 : Les douze collocations Mot1-Mot2 les plus fréquentes en DAE (effectifs et proportions)

\begin{tabular}{|l|l|l|}
\hline Collocations Mot1-Mot2 & Effectifs (effectifs cumulées) & Proportions (proportions cumulées) \\
\hline en a(s) & $124(124)$ & $10,7 \%(10,7 \%)$ \\
\hline peut-être & $57(181)$ & $4,7 \%(15,4 \%)$ \\
\hline vas-y & $57(238)$ & $4,7 \%(20,1 \%)$ \\
\hline tout à $+X^{7}$ & $32(270)$ & $2,6 \%(22,7 \%)$ \\
\hline on a & $27(297)$ & $2,2 \%(25 \%)$ \\
\hline en ai & $25(322)$ & $2,1 \%(27 \%)$ \\
\hline ils ont & $25(347)$ & $2,1 \%(29,1 \%)$ \\
\hline vous avez & $23(370)$ & $1,9 \%(31 \%)$ \\
\hline en av(ait/aient/ais/ez) & $23(393)$ & $1,9 \%(32,9 \%)$ \\
\hline un autre & $21(414)$ & $1,7 \%(34,7 \%)$ \\
\hline c'est un & $20(434)$ & $1,7 \%(36,3 \%)$ \\
\hline on en & $16(450)$ & $1,3 \%(37,6 \%)$ \\
\hline
\end{tabular}

\footnotetext{
${ }^{4}$ La méthodologie mise en place pour le projet ALIPE nous a permis de distinguer deux types d'énoncés adultes : ceux produits en direction de l'enfant et ceux produits en direction d'un adulte. Les données du DAE ne concernent donc que ce premier type d'énoncés, desquels sont extraites 1.209 liaisons réalisées.

${ }^{5}$ À savoir « en a(s) », « vas-y », « on a », « en ai », « ils ont » et « vous avez ».

${ }^{6}$ Ainsi, plus l'indice est élevé et moins les Mots 2 utilisés sont diversifiés.

${ }^{7}$ Ce cadre lexical de liaison regroupe des expressions figées comme «tout à l'heure », « tout à fait » ou « tout à coup » par exemple.
} 
Author's Pre-print: Liégeois, L. (2017). Acquisition des liaisons nominales et verbales : de la lexicalisation à l'abstraction des constructions. Langue Française, 194(2). pp. 125-146. doi:10.3917/lf.194.0125.

\begin{tabular}{|l|l|l|}
\hline TOTAL & 249 collocations Mot1-Mot2 différentes & 1.209 liaisons réalisées
\end{tabular}

Tableau 5 : Indices de diversité lexicale des contextes de liaison en DAE. Tableau adapté de Liégeois (2014)

\begin{tabular}{|l|l|l|l|l|l|}
\cline { 3 - 6 } & \multirow{2}{*}{ Mots1 } & \multicolumn{2}{l|}{ Parents de Salomé } & \multicolumn{2}{l|}{ Parents de Prune } \\
\cline { 3 - 7 } & & Nb. de Mots1 & Rapport Mot1/Mot2 & Nb. de Mots1 & Rapport Mot1/Mot2 \\
\hline \multirow{4}{*}{ Mots1 en liaison nominale } & des & 25 & 1,9 & 59 & 2 \\
\cline { 2 - 7 } & les & 83 & 1,5 & 87 & 1,7 \\
\cline { 2 - 7 } & un & 34 & 1,6 & 100 & 2,1 \\
\hline \multirow{4}{*}{ Mots1 en liaison verbale } & en & 117 & 5,6 & 180 & 7,9 \\
\cline { 2 - 7 } & ils & $/$ & $/$ & 30 & 5 \\
\cline { 2 - 7 } & on & 77 & 4,3 & 124 & 3,5 \\
\cline { 2 - 6 } & vous & $/$ & $/$ & 64 & 3,8 \\
\hline
\end{tabular}

\section{DISCUSSION}

À partir du troisième stade du scénario lexical de l'acquisition de la liaison nominale, les données de notre corpus atteste de l'apparition d'erreurs inexistantes aux stades précédents. Il s'agit d'erreurs concernant des formes verbales, qui représentent près de $21 \%$ des erreurs d'adjonction produites par Prune au T1. Ces erreurs résulteraient de la compétition entre deux types de constructions: les constructions lexicalisées et les constructions abstraites. Nous pouvons avancer deux arguments empiriques soutenant l'hypothèse que la plupart des contextes verbaux de liaison catégorique sont mémorisés sous la forme de constructions lexicalisées :

1. Les Mots1 employés en contexte verbal en DAE sont suivis d'un nombre limité de Mots2, ce qui favoriserait la mémorisation de constructions lexicalisées.

2. À un stade précoce, nous ne relevons aucune erreur en contexte verbal due à un remplacement ou à une omission de la CL. Ces résultats corroborent ceux de Dugua (2006) qui ne relève aucune erreur de liaison entre un pronom et un verbe dans un corpus de 898 erreurs relevées au vol.

L'enfant aurait ainsi mémorisé majoritairement, au cours des deux premiers stades du scénario lexical, des contextes de liaison verbale sous la forme d'une construction figée intégrant la CL. Confronté à davantage de collocations de type $|\mathrm{PRO}+\mathrm{V}|$, l'enfant va ensuite être amené, comme plus tôt en contexte nominal, à repérer progressivement l'analogie entre les différentes collocations rencontrées en input. En effet, le lexique utilisé en DAE se diversifie au cours du développement, entraînant ainsi une multiplication des formes de Mots2 et une baisse du rapport Mot1/Mots2 en contexte verbal entre T1 et T2 (Liégeois, 2014). Cet accroissement de la fréquence des types de liaison verbale favoriserait l'émergence de constructions plus abstraites et entraînerait, sur la base du schéma standard de la syllabe en CV, une segmentation progressive des constructions verbales lexicalisées.

Il découlerait de ce découpage la mémorisation d'exemplaires comme $\mid z$-ont $\mid$ ou $\mid z$-étaient $\mid$. Ces exemplaires verbaux, comme pour les noms, pourraient ensuite être insérés dans un slot disponible pour une forme verbale. Il en résulte donc des erreurs d'adjonction comme celles que nous avons observées dans les productions de Prune (cf. Extrait de corpus 1$)^{8}$. Par exemple, la fillette insèrerait l'exemplaire $\mid z$-ont $\mid$ dans le slot disponible pour un verbe dans une construction de type $\mid \mathrm{N}+n e+\mathrm{V}+$ pas $\mid$.

Cette hypothèse se trouve renforcée par la grande fréquence d'emploi de la CL /z/ avant les formes verbales «ont» et «étaient ». En nous référant aux données de productions parentales du corpus de Paris ${ }^{9}$ (Morgenstern \& Parisse, 2012), nous notons que $77,7 \%$ des emplois de la forme « ont » (302/292) se trouvent précédés des pronoms personnels «ils » ou « elles », entraînant la réalisation de la CL /z/. Concernant la forme à l'imparfait «étaient», la situation est identique pour 78,9\% des cas. Si, comme le postule la TUC, la fréquence d'usage d'un exemplaire renforce son ancrage cognitif en mémoire, alors il est tout à fait logique que la majorité des quelques erreurs que nous avons pu relever concernent les formes $\mid z$-ont $\mid$ et $\mid z$-étaient $\mid$, particulièrement fréquentes dans les énoncés adressés aux enfants.

Extrait de corpus $1:$ Exemples d'erreur impliquant des exemplaires de formes verbales

$[\ldots]$

CHI-Prune [u1608-ali-prune-071120-1] : et les mammouths ne -Z- ont pas de zizi .

FAT-Prune [u1609-ali-prune-071120-1] : ah d'accord .

CHI-Prune [u1610-ali-prune-071120-1] : qui est-c(e) les plus forts ?

FAT-Prune [u1611-ali-prune-071120-1] : alors eux c'est qui ?

\footnotetext{
${ }^{8}$ Pour une écoute en streaming de l'extrait, suivre le lien suivant : https://hdl.handle.net/11403/alipe000853/extraits sonores/ali-prune-071120-1 3327299-3349330.mp3

${ }^{9} \mathrm{Ce}$ corpus, disponible dans la banque de corpus CHILDES, se compose d'interactions parents-enfants.
} 
Author's Pre-print: Liégeois, L. (2017). Acquisition des liaisons nominales et verbales : de la lexicalisation à l'abstraction des constructions. Langue Française, 194(2). pp. 125-146. doi:10.3917/1f.194.0125.

CHI-Prune [u1612-ali-prune-071120-1] : c'est les ceux ceux qui -Z- ont des des des zizis !

Notre méthodologie de recueil des données nous a permis de nous pencher sur des contextes qui ont reçu, jusqu'à maintenant, très peu d'attention de la part des chercheurs. De plus, nos données semblent conforter le scénario fondé sur les postulats de la TUC proposé pour les liaisons nominales, dans le sens où des processus identiques semblent à l'œuvre pour le contexte verbal, confirmant donc l'hypothèse de Chevrot et al. (2007). Il apparaît simplement que les processus de segmentation des constructions, d'abstraction et de mémorisation des exemplaires se produisent plus tardivement. Comme nous l'avons montré pour l'acquisition de la liaison nominale (Liégeois, 2014), cette asymétrie peut être expliquée en prenant en considération des facteurs d'usage. Il apparaît en effet que les contextes de liaison verbale sont davantage figés en input, dans le sens où les pronoms personnels sélectionnent un nombre beaucoup moins important de formes de Mots2 que les déterminants. Ainsi, l'enfant aurait à sa disposition un nombre très limité de collocations Mot1-Mot2 différentes sur lesquelles s'appuyer pour procéder à une analogie fondée sur la forme linguistique, ce qui aboutirait donc à une abstraction plus tardive des constructions comportant une liaison verbale.

Les résultats et les hypothèses présentés ici, fondés sur les productions d'un nombre limité de sujets, sont amenés à être approfondis et testés sur un échantillon plus important de sujets. C'est notamment l'objectif des expérimentations mises en place par Dugua et al. (2017). 
Author's Pre-print: Liégeois, L. (2017). Acquisition des liaisons nominales et verbales : de la lexicalisation à l'abstraction des constructions. Langue Française, 194(2). pp. 125-146. doi:10.3917/lf.194.0125.

\section{Références}

Аввот-Sмith, K. \& Tomasello, M. (2006), "Exemplar-learning and schematization in a usage-based account of syntactic acquisition", The Linguistic Review 23(3), 275-290.

BeHRENS, H. (2009), "Usage-based and emergentist approaches to language acquisition", Linguistics 47(2), 383-411. [http://doi.org/10.1515/LING.2009.014]

Bybee, J. (2001), "Frequency effects on French liaison", in J. BYBEe \& P. J. HOPPER (eds.), Frequency and the Emergence of Linguistics Structures, Amsterdam/Philadelphia: John Benjamins Publishing Company, 337-359.

BYBeE, J. (2005), « La Liaison : effets de fréquence et constructions », Langages 39, 24-37.

BYBEe, J. (2006), "From usage to grammar: The mind's response to repetition", Language 82(4), 711-733.

Bybee, J. (2010), Language, Usage and Cognition, Cambridge: Cambridge University Press.

Bybee, J. \& Hopper, P. J. (2001), "Introduction", in J. Bybee \& P. J. Hopper (eds.), Frequency and the Emergence of Linguistics Structures, Amsterdam/Philadelphia: John Benjamins Publishing Company, 1-24.

Chevrot, J.-P., Chabanal, D. \& Dugua, C. (2007), « Pour un modèle de l'acquisition des liaisons basé sur l'usage : trois études de cas », Journal of French Language Studies 17(1), 103-128. [http://doi.org/10.1017/S0959269506002663]

Chevrot, J.-P., Dugua, C. \& Fayol, M. (2005), «Liaison et formation des mots en français: un scénario développemental », Langages 158, 38-52.

Chevrot, J.-P., Dugua, C. \& Fayol, M. (2009), "Liaison acquisition, word segmentation and construction in French: a usage based account", Journal of Child Language 36(3), 557-596. [http://doi.org/10.1017/S0305000908009124]

Chevrot, J.-P., Dugua, C., Harnois-Delpiano, M., Siccardi, A. \& Spinelli, E. (2013), "Liaison acquisition: debates, critical issues, future research", Language Sciences 39, 83-94. [http://doi.org/10.1016/j.langsci.2013.02.011]

Cоте, M.-H. (2005), «Le statut lexical des consonnes de liaison», Langages 39(158), 66-78. [http://doi.org/10.3406/lgge.2005.2663]

Croft, W. \& Cruse, A. (2004), Cognitive Linguistics. Cambridge: Cambridge University Press.

Diessel, H. (2013), "Construction Grammar and First Language Acquisition", in T. Hoffmann \& G. Trousdale (eds.), The Oxford Handbook of Construction Grammar, Oxford: Oxford University Press, 347-363. [http://doi.org/10.1093/oxfordhb/9780195396683.013.0019]

Dugua, C. (2006), Liaison, segmentation lexicale et schémas syntaxiques entre 2 et 6 ans. Un modèle développemental basé sur l'usage, Thèse de doctorat, Université Stendhal - Grenoble III.

Dugua, C., Nardy, A., Liegeois, L., Chevrot, J.-P., Chabanal, D. (2017). «L'acquisition des liaisons après les clitiques préverbaux est-elle spécifique? Apport d'une expérimentation à grande échelle ». Journal of French Language Studies 27(1), 73-86.

ENCREVE, P. (1988), La liaison avec et sans enchaînement. Paris : Édition du Seuil.

FranÇOIS, J. (2008), «Les grammaires de construction : un bâtiment ouvert aux quatre vents », Cahiers Du CRISCO 26, 119.

FrançoIs, J. (2011), «Construction et exemplaires. Une nouvelle approche des structures prédicatives du français illustrée par la configuration [N s'en V] », Philologie Im Netz, 58, 19-38.

Goldberg, A. E. (1995), Constructions: A Construction Grammar Approach to Argument Structure. Chicago: University of Chicago Press.

Goldberg, A. E. (2006), Constructions at Work: The Nature of Generalization in Language. Oxford: Oxford University Press.

Gries, S. T. (2013). "Data in Construction Grammar". In T. Hoffmann \& G. Trousdale (eds.), The Oxford Handbook of Construction Grammar, Oxford University Press, 93-108. [http://doi.org/10.1093/oxfordhb/9780195396683.013.0006]

Kemmer, S. \& Barlow, M. (2000), "Introduction: A Usage-Based Conception of Language", In M. BARLOW \& S. Kemmer (eds.), Usage-based models of language, Sanford Californie: CSLI Publications, VII-XXVIII.

LANGACKER, R. W. (1986), "An Introduction to Cognitive Grammar", Cognitive Science, 10(1), 1-40. [http://doi.org/10.1016/S0364-0213(86)80007-6]

Langacker, R. W. (1987), Foundations of Cognitive Grammar. Volume I: Theoretical Prerequisites. Stanford: Stanford University Press.

LANGACKeR, R. W. (2008), Cognitive Grammar: A Basic Introduction. New-York: Oxford University Press.

LANGACKER, R. W. (2009), "A dynamic view of usage and language acquisition". Cognitive Linguistics, 20(3), 627-640. [http://doi.org/10.1515/COGL.2009.027]

Leroy, S., Parisse, C. \& Maillart, C. (2009), «Les difficultés morphosyntaxiques des enfants présentant des troubles spécifiques du langage oral : une approche constructiviste », Rééducation Orthophonique, 238, 21-45.

LiEgEOIS, L. (2013). « De l'analyse au partage des données, quel(s) format(s) choisir? L'exemple d'un corpus d'interactions parents-enfant ». In M. Damiani, K. Dolar, C. Florez-Pulido, R. Loth, J. Magnier, \& A. Pegaz (eds.), Traitement de corpus (Actes de Coldoc 2012), Paris: Modyco, 128-142.

LIEGEOIS, L. (2014), Usage des variables phonologiques dans un corpus d'interactions naturelles parents-enfant: impact du bain linguistique et dispositifs cognitifs d'apprentissage. Thèse de doctorat, Université Blaise Pascal. 
Author's Pre-print: Liégeois, L. (2017). Acquisition des liaisons nominales et verbales : de la lexicalisation à l'abstraction des constructions. Langue Française, 194(2). pp. 125-146.

doi:10.3917/1f.194.0125.

Liegeois, L., Chanier, T. \& Chabanal, D. (2014). Corpus globaux ALIPE : Interactions parents-enfant annotées pour l'étude de la liaison, Nancy : Ortolang, [http://hdl.handle.net/11041/alipe-000853].

MacWhinneY, B. (2000). The CHILDES Project: Tools for Analyzing Talk (3rd Edition), Mahwah, NJ: Lawrence Erlbaum Associates.

Matthews, D., Lieven, E., Theakston, A. \& Tomasello, M. (2005), "The role of frequency in the acquisition of English word order", Cognitive Development, 20(1), 121-136. [http://doi.org/10.1016/j.cogdev.2004.08.001]

Morgenstern, A. \& Parisse, C. (2012), "The Paris Corpus", Journal of French Language Studies, 22(1), 7-12. [http://doi.org/10.1017/S095926951100055X]

NARDY, A. (2008), Acquisition des variables sociolinguistiques entre 2 et 6 ans: facteurs sociologiques et influences des interactions au sein du réseau social, Thèse de doctorat, Université Stendhal - Grenoble III.

Pierrehumbert, J. (2001), "Exemplar dynamics: Word frequency, lenition and contrast", in J. L. Bybee \& P. Hopper (eds.), Frequency and the Emergence of Linguistics Structures, Amsterdam/Philadelphia: John Benjamins Publishing Company, $137-158$.

PINKER, S. (1999), Words and Rules: The Ingredients of Language, New-York: Basic Books.

SicCARDi, A. (2015), Unités et séquences dans le lexique adulte et enfantin, Université Stendhal - Grenoble III.

Tomasello, M. (2003), Constructing a Language: A Usage-Based Theory of Language Acquisition, Cambridge: Harvard University Press.

WAUQuier-Gravelines, S. \& Braud, V. (2005), «Proto-déterminant et acquisition de la liaison obligatoire en français », Langages, 39(158), 53-65. [http://doi.org/10.3406/lgge.2005.2662]

WAUQuier, S. (2009), "Acquisition de la liaison en L1 et L2: stratégies phonologiques ou lexicales?», Acquisition et Interaction en Langue Étrangère, 2, 93-130.

Wauquier, S. \& Shoemaker, E. (2013), "Convergence and divergence in the acquisition of French liaison by native and non-native speakers: a review of existing data and avenues for future research", Language, Interaction et Acquisition, 4(2), 161-189. 